\title{
Proposta de modelo de análise e otimização de processos para o setor de serviços visando a melhoria da qualidade
}

Izabel Alinne Alves de Paula email_izabelalinne@hotmail.com

\begin{abstract}
RESUMO
A literatura aponta diversas ferramentas capazes de avaliar e melhorar a qualidade em serviços, entretanto predominam-se mecanismos subjetivos, que se resumem a mensurar a satisfação do cliente ao fim do processo. É certo que, a qualidade tem foco no cliente, mas na atual Era da Qualidade assume-se que ela é gerada durante o processo produtivo. Daí objetivou-se construir um modelo de análise e melhoria de processo, respaldado nos preceitos da Gestão da Qualidade, capaz de otimizar processos executados no setor de serviços, através do uso de técnicas de gestão e estatística, como também gerasse dados objetivos para que assim a tomada de decisão fosse feita de forma mais robusta. Para isto, desenvolveu-se uma pesquisa de cunho teórico-conceitual, de aspecto exploratório, qualitativo e de natureza básica. Ao fim, através da concatenação das fases do Método de Análise e Solução de Problemas (MASP) com as etapas de Planejamento Experimental foi obtido modelo.
\end{abstract}

PALAVRAS-CHAVE: Modelo. Qualidade. Processo. Serviço. 


\section{INTRODUÇÃO}

Muitos autores caracterizam a qualidade como um diferencial competitivo, que viabiliza a conquista do cliente e a sua fidelidade ao produto ou serviço, mas já há também quem defenda que a qualidade não é só um diferencial, ela é uma obrigação para o sucesso e sobrevivência da organização (BATALHA, 2008; CARPINETTI, 2010; PEINADO; GRAEML, 2007; RAMSEOOK-MUNHURRUN; LUKEABHIWAJEE; NAIDOO, 2010).

Montgomery (2009) afirma que "qualidade é inversamente proporcional à variabilidade" e que a sua melhoria "é a redução da variabilidade nos processos e produtos", onde a variabilidade "só pode ser descrita em termos estatísticos". Dando a entender que para se promover a qualidade, de fato, é necessário utilizar métodos estatísticos para controlar os fatores do processo. Quanto a esta variação Rodríguez e Franco (2009) afirmam que embora as causas da variação na qualidade sejam inumeráveis, nem todas afetam o processo com a mesma intensidade.

Analisando os recursos estatísticos que garantem promover a qualidade, identificou-se que, ainda que estas ferramentas estatísticas sejam genéricas, predominantemente são aplicadas em ambientes de manufatura (ANTONY et al., 2010), percebeu-se também que para o setor de serviço existe uma gama de ferramentas específicas, contudo, observam-se diferenças significativas entre estes tipos de ferramentas que normalmente apreciam apenas a satisfação do cliente ao final da prestação do serviço, através da inquirição de fatores categóricos, subjetivos a opinião do indivíduo, enquanto aquelas ferramentas estatísticas priorizam analisar se no processo produtivo há o resguardo das características da qualidade. É certo que a qualidade tem foco na satisfação do cliente, mas é fato que ela é gerada no processo produtivo.

Considerando estes argumentos, esse trabalho teve como objetivo propor um modelo de análise e melhoria de processos que respaldado em técnicas estatísticas fosse capaz de otimizar processos executados no setor de serviços, com foco na melhoria da qualidade.

$\mathrm{Na}$ realização deste trabalho observou-se uma escassez de estudos mais objetivos acerca dos processos do setor de serviço, viu-se que neste campo se faz necessário implantar modelos que contemplem as especificidades dos serviços e que gerem dados lógicos para conduzir a tomada de decisão de forma mais confiável.

\section{REFERENCIAL TEÓRICO}

Esta seção apresenta conceitos sobre qualidade e processos do setor de serviço, os quais foram considerados durante o processo de construção do modelo de melhoria e otimização de processos.

\section{QUALIDADE EM SERVIÇOS}

Qualidade é uma das palavras-chaves mais difundidas junto à sociedade e nas empresas (CARPINETTI, 2010). No entanto, a sua essência variou bastante 
desde a Revolução Industrial (iniciada na Inglaterra em meados do século XVIII) até os dias atuais, observa-se que a ênfase mudou do produto para o processo, tanto que na atual Era da Qualidade - Gestão da Qualidade, a ideia é que a qualidade é gerada no processo produtivo (CORRÊA; CORRÊA, 2004; PALADINI, 1995); neste viés, Forno (2005) acrescenta que essa evolução da qualidade ocorreu essencialmente no contexto do setor de manufatura, ficando o setor de serviço, a princípio, a margem desse processo.

Estudiosos afirmam que definir a palavra qualidade é uma tarefa complexa e de difícil consenso, devido à percepção subjetiva adotada por cada indivíduo, ou seja, a qualidade é uma entidade multifacetada (ARAUJO; PAULA; SILVA, 2012; BATALHA, 2008; CARPINETTI, 2010; MONTGOMERY, 2009; VALLS, 2005).

Difundem-se conceitos de qualidade, baseados nas teorias geradas por alguns estudiosos que tiveram maior influência nesta área, os chamados 'gurus da qualidade'. Mas o que é qualidade? De maneira geral, ainda que a definição de qualidade seja particular, há um entendimento dominante nas últimas décadas, o qual a qualidade é um atributo que está relacionado à adequação do uso e a conformidade das especificações do produto (CARPINETTI, 2010; RODRÍGUEZ; FRANCO, 2009; VALLS, 2005), sendo que "o produto é o resultado do processo" (PALADINI, 1995, p. 18).

O trabalho desenvolvido por Parasuraman, Zeithaml e Berry (1985), a Escala SERVQUAL, é um dos mais citados quando se busca sobre avaliação da qualidade de serviços, de acordo com eles a qualidade na prestação de serviços pode ser medida pela comparação da percepção do serviço prestado com as expectativas do serviço desejado. Entretanto, muitas são as críticas feitas a este modelo, dentre elas destaca-se a afirmativa que com ele é possível medir a satisfação do cliente, não a qualidade do serviço (RIBEIRO, 2010; SIQUEIRA, 2006; SOUSA et al., 2011), diante disto surgiram diversos outros modelos, que utilizam dimensões específicas para a análise da qualidade em serviços.

Quanto a atual era da qualidade, Carpinetti (2010, p. 17) declara que foi a partir da década de 1950 que a "gestão da qualidade ganhou uma nova dimensão, expandindo-se para as etapas mais a montante e a jusante do ciclo de produção, envolvendo toda a organização". Considerando então que nesta era o foco da qualidade é o processo, ressalta-se a Gestão da Qualidade no Processo, que segundo Paladini (1995, p. 18) este modelo de gestão direciona "todas as ações do processo produtivo para o pleno atendimento do cliente", isto sendo feito por meio da melhoria organizacional do processo, em três etapas: a eliminação de perdas; a eliminação das causas das perdas e a otimização do processo. A natureza permanente desta última etapa caracteriza a melhoria contínua, sendo isto uma ideia típica da Gestão da Qualidade Total (TQM) que é entendida como "uma filosofia de permanente melhoria em tudo que fazemos" (JUNYENT et al., 2009, p. 93).

Quando se fala de gestão de processos, logo o Ciclo PDCA se destaca, pois é o método gerencial mais difundido nas literaturas de gestão (BATALHA, 2008; CARPINETTI, 2010; PEINADO; GRAEML, 2007; WERKEMA; AGUIAR, 1996a). O Ciclo PDCA pode ser concebido como a consolidação dos princípios básicos da Gestão da Qualidade, pois ele viabiliza a visão sistêmica do processo para a melhoria contínua. 
Considerando que o foco desta pesquisa é a melhoria da qualidade em processos, é válido destacar as etapas do MASP (Quality Control Story, QC-Story), conforme Figura1.

Figura 1 - Ciclo PDCA utilizado para o alcance das metas de melhoria

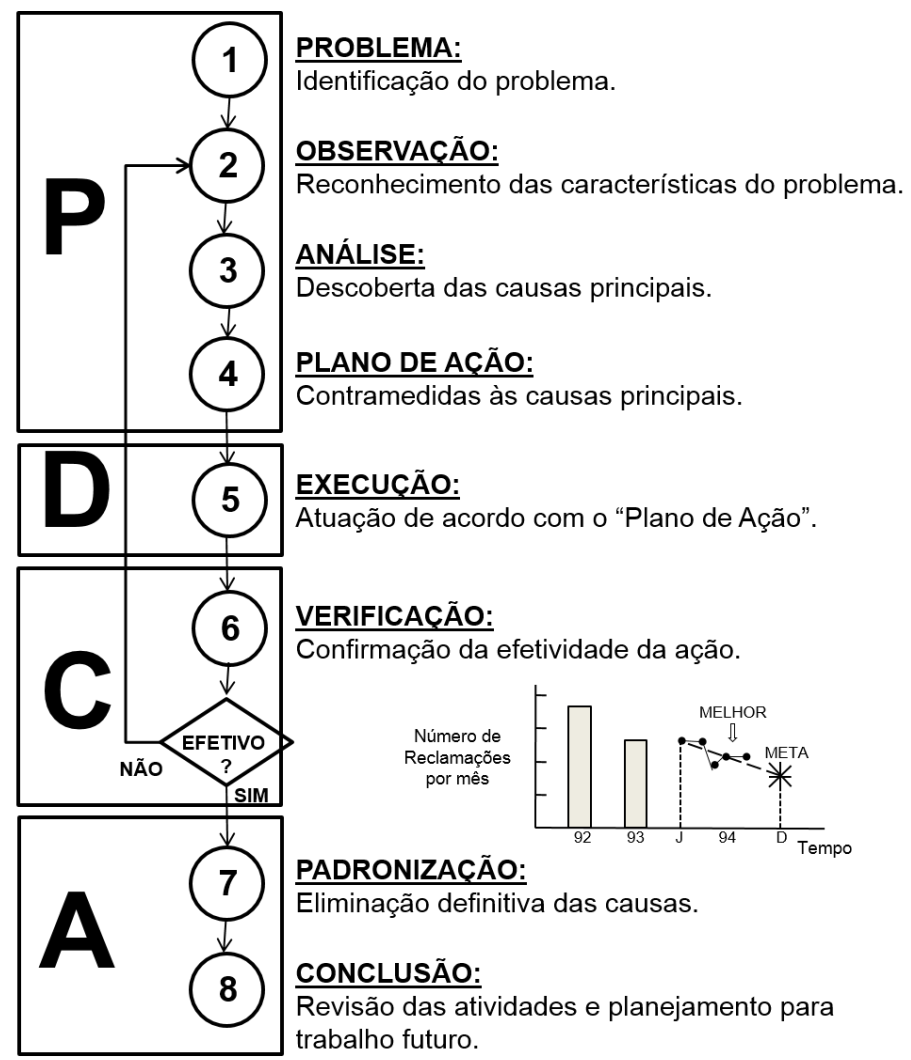

Fonte: Campos (1994) apud Werkema e Aguiar (1996a)

- Identificação do problema: busca-se pelas dificuldades mais críticas, e então são eleitos os problemas prioritários a serem corrigidos;

- Observação: faz-se o reconhecimento das características do problema;

- Análise: investigam-se as causas raízes do problema;

- Plano de ação: nesta fase, elabora-se um plano de ação para eliminar ou mitigar os efeitos indesejáveis;

- Ação: implantação do plano de ação;

- Verificação: consiste da avaliação dos resultados a fim de verificar se as ações foram eficazes para resolver ou minimizar os problemas. Em caso positivo, segue-se para a próxima fase, caso contrário, reiniciarse o processo a partir da Observação;

- Padronização: faz-se a inserção das ações implementadas na rotina de operação do processo e,

- Conclusão: finaliza-se o processo com o registro de todas as ações exploradas. 
Carpinetti (2010) aponta que uma característica marcante deste processo de melhoria é o uso da abordagem científica, onde o processo de tomada de decisão é fundamentado em dados e fatos oriundos de atividades logicamente sequenciadas e não de 'achismo'. Assim, para cada fase do ciclo são indicadas ferramentas para a coleta, o processamento e a disposição dos dados (ARAUJO; PAULA; SILVA, 2012; WERKEMA; AGUIAR, 1996a).

As ferramentas que ajudam na implantação da qualidade no processo são abundantes, mas segundo Corrêa e Corrêa (2004, p. 212) elas por si só não resolvem os conflitos, mas "apoiam e auxiliam pessoas na tomada das decisões que resolverão problemas e melhorarão situações". Dividem-se em três grupos as ferramentas para a gestão da qualidade no processo: ferramentas tradicionais, classificadas como As sete ferramentas da qualidade, que procuram conhecer o processo e então melhorá-lo; novas ferramentas, conhecida como As sete ferramentas gerenciais, que buscam aperfeiçoar o processo, isto é, subsidiam a própria melhoria e, outras ferramentas, que visam organizar o processo e logo a melhoria se desenvolve por consequência.

\section{PROCESSOS DO SETOR DE SERVIÇO E SUA OTIMIZAÇÃO}

O setor de serviços tem ganhado maior importância no setor econômico ao longo dos anos (FORNO, 2005; PRASS; SANT'ANNA; GODOY, 2010). Ainda assim, o setor de serviço é "um dos setores mais mal definidos [...] e é efetivamente um dos campos mais delicados a serem explorados, pois suas próprias fronteiras são problemáticas" (SIQUEIRA, 2006, p. 36).

Busca-se conhecer a essência de serviços pelas características (dimensões) específicas de suas operações (CARVALHO; BRITO; CABRAL, 2010; COLLIER; MEYER, 1998; CORRÊA; CORRÊA, 2004; GIANESI; CORRÊA, 2011; SIQUEIRA, 2006). As características básicas são: intangibilidade, necessidade da presença do cliente ou um bem de sua propriedade, além da produção e consumo simultâneos.

Considerando estes atributos, concebe-se que serviço é uma atividade de natureza intangível, que ocorre durante a interação entre cliente (ou bem de sua propriedade) e o prestador de serviço (recurso humano ou físico), buscando a solução do(s) problema(s) do cliente.

Há algumas décadas, devido à necessidade de um maior aperfeiçoamento na gestão das operações de serviços, tem-se procurado adaptar as técnicas da gestão industrial, desenvolvidas a princípio para o setor de manufatura. Sendo que a preocupação em tratar o setor de serviços com maior seriedade, não tem foco somente em aumentar a produtividade, existe também à preocupação com a satisfação do cliente (GIANESI; CORRÊA, 2011).

Pode-se afirmar que processo é uma combinação de equipamentos, informações do processo, métodos, pessoas e condições ambientais, o qual transforma alguma entrada em uma saída, que apresenta uma ou mais respostas observáveis. Contudo, todo processo sofre influência de variáveis, umas controláveis, outras não (LIMA, 2006; MONTGOMERY, 2009; WERKEMA; AGUIAR, 1996b), conforme observado na Figura 2. 
Figura 2 - Entradas e saídas de um processo de produção

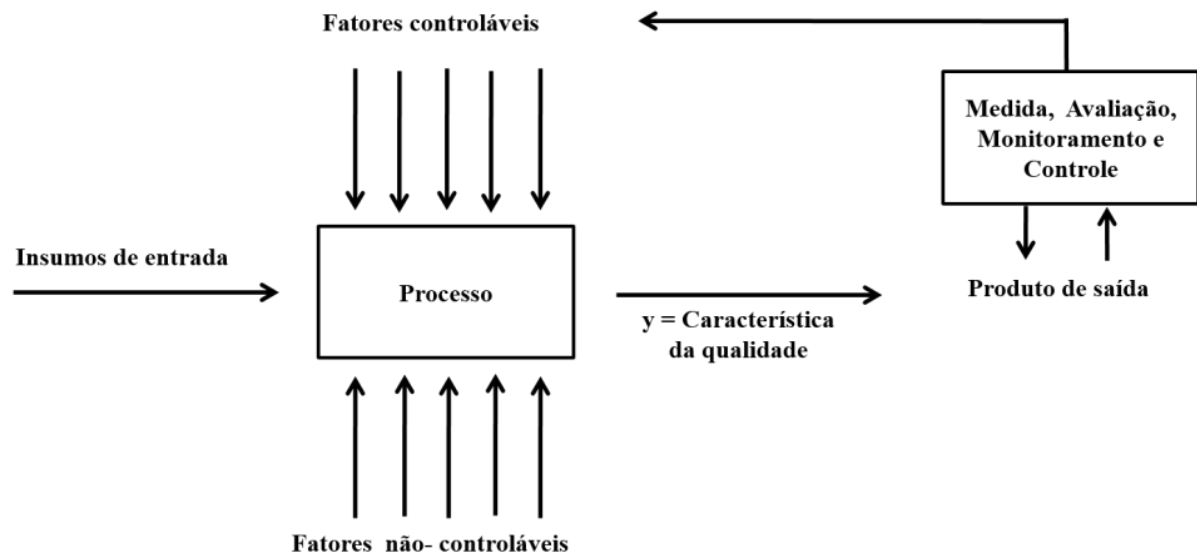

Fonte: Autoria própria (2013)

Assim, observa-se que o setor de serviço é complexo por sua natureza imaterial e também porque na sua produção não existe a possibilidade de separar com nitidez o processo produtivo da prestação de serviço, ambos se confundem (ANTONY et al., 2010; COLLIER; MEYER, 1998; CORRÊA; CORRÊA, 2004; JUNYENT et al., 2009).

Através de uma abordagem científica pode-se compreender melhor os processos de serviços e o comportamento dos clientes e isto faz mudar uma cultura de decisões que são baseadas em apenas sentimentos, passando então a serem baseada em fatos (ANTONY et al., 2010; LIMA, 2006; WERKEMA; AGUIAR, 1996b). Assim, considera-se oExperimental uma importante ferramenta a ser seguida durante a busca da otimização de processos e melhoria da qualidade.

O Planejamento Experimental (em inglês Design of Experiments, DOE) é uma técnica utilizada para identificar e avaliar os principais fatores que influenciam os itens de controle de um processo, simultaneamente (MONTGOMERY, 2009). Antony et al. (2010) afirmam que esse instrumento é fundamental e crucial para aumentar a compreensão de um produto ou processo, pois ele fornece um meio poderoso para alcançar melhorias na qualidade do produto e eficiência do processo.

Considerando o planejamento da qualidade, Werkema e Aguiar (1996b) afirmam que este planejamento deve ser desenvolvido para estabelecer novos padrões de trabalho baseados nas necessidades das pessoas, sejam clientes internos ou externos. Lima (2006) declara que a aplicação das técnicas de Planejamento Experimental em processos pode: melhorar rendimento do processo, minimizar a variabilidade do processo, introduzir a padronização, reduzir os tempos de projeto e desenvolvimento, além de diminuir os custos de operação.

Galdámez (2002) aponta que antes de iniciar qualquer experimentação, é necessário estabelecer o planejamento dos testes. Lima (2006), Montgomery (2009), Werkema e Aguiar (1996a) e Wu e Hamada (2009) fazem referência a um roteiro para planejar um experimento. Considerando que de modo geral as etapas são semelhantes ou se completam, a seguir são descritas em sete etapas as recomendações propostas: 
a) reconhecimento do problema e identificação dos objetivos do experimento: feito através do conhecimento e experiência de quem lida diretamente com os processos;

b) seleção de uma variável resposta: deve-se escolher uma variável resposta que de fato forneça informação útil sobre o processo em estudo; múltiplas respostas não são comuns;

c)escolha dos fatores e níveis a serem variados no experimento: utilizam-se conhecimentos não estatísticos (prévio conhecimento) para apontar os fatores que irão variar no experimento e os que irão permanecer constantes assim como a faixa de variação. Em geral, a escolha dos fatores e a seleção da variável resposta são feitas simultaneamente ou podem ser feitas em ordem inversa;

d) planejamento e escolha do tipo de procedimento experimental: a escolha do planejamento envolve a consideração sobre as possíveis interações entre os fatores, as influências ambientais sobre o experimento (tempo, fator climático, mão-de-obra, equipamento, etc.), o tamanho da amostra e o método de aleatorização;

e) execução do experimento: realizam-se os procedimentos conforme foi planejado anteriormente, monitorando o progresso do experimento e registrando os dados que forem apresentados;

f) análise de dados: executa-se um processo de revisão dos dados, com o objetivo de verificar erros de registro, em seguida, faz-se a análise crítica dos dados obtidos, isto podendo ser feito através de métodos gráficos ou estatísticos;

g) conclusões: interpretação dos resultados e elaboração de relatórios, considera-se todos os dados coletados no experimento, mostra-se os gráficos e detalha-se os dados numericamente para tornar clara a interpretação dos resultados, deve-se registrar tudo inclusive as possíveis limitações impostas pelos dados ou métodos e também deve-se mostrar a importância do problema tratado e o significado práticos dos resultados obtidos.

$\mathrm{Na}$ realização de um bom experimento deve-se fazer uso de planos estruturados de trabalho para que o desenvolvimento da pesquisa fique delineado ao objetivo proposto. Métodos estatísticos podem aumentar a eficiência dos experimentos e fortalecer as conclusões obtidas (LIMA, 2006). Assim, na fase de planejamento e escolha do tipo de procedimento deve-se analisar e verificar as técnicas experimentais, disponíveis na literatura, que melhor admitam as características dos dados em estudo, sejam eles numéricos ou categóricos, pois isto implica na validação dos resultados.

\section{ASPECTOS METODOLÓGICOS}

Inicialmente realizou-se uma pesquisa bibliográfica "desenvolvida com base em material já elaborado" (GIL, 2002). Buscou-se identificar estudos relevantes sobre qualidade no setor de serviços, como também métodos gerenciais, científicos e estatísticos que poderiam ser utilizados na determinação da região ótima de processos.

Para atender o objetivo deste estudo foi desenvolvida uma pesquisa exploratória, que em "síntese visa à inovação pela proposição de novos modelos" 
(JUNG, 2010). Assim, com o propósito de adaptar um modelo que fosse capaz de analisar e viabilizar a melhoria da qualidade em processo mesclaram-se as etapas do MASP, que fez garantir o caráter gerencial, com as etapas do roteiro de Planejamento Experimental, que assegurou o viés científico e objetivo. Pelo fato destes dados serem de natureza descritiva e tendo sido eles analisados indutivamente (CERVO; BERVIAN; SILVA, 2007; SILVA; MENEZES, 2005), definiu-se como qualitativa a abordagem da pesquisa (GIL, 2002; JUNG, 2010).

Definiu-se este trabalho como de cunho teórico-conceitual, que segundo Miguel (2010) "seu escopo principal envolve, sobretudo, modelagens conceituais que resultam em novas teorias". Considerou a natureza da pesquisa como básica, que segundo Jung (2010) tem como objetivo descrever fenômenos e divulgar o conhecimento.

Destaca-se que essa pesquisa é o produto de uma pesquisa de mestrado, que foi validado por um aplicação em um conjunto de processos (PAULA; SILVA, 2016).

\section{PROPOSTA DE MODELO DE ANÁLISE E MELHORIA DE PROCESSOS AO SETOR DE SERVIÇO}

O uso do Ciclo PDCA é inquestionável no gerenciamento de processos (ARAUJO; PAULA; SILVA, 2012; BATALHA, 2008; CARPINETTI, 2010; CORRÊA; CORRÊA, 2004; PEINADO; GRAEML, 2007; WERKEMA; AGUIAR, 1996a, 1996b) e, com o objetivo de construir um modelo pautado na abordagem de melhoria de processos, respeitaram-se as oito fases do modelo MASP/PDCA.

Para a condução da otimização de processos, a literatura (LIMA, 2006; MONTGOMERY, 2009; WERKEMA; AGUIAR, 1996a; WU; HAMADA, 2009) indica que seja feito o Planejamento de Experimentos, desta forma, procurou-se construir um modelo guiado pelas etapas desta metodologia.

Concatenando as etapas dos modelos supramencionados, construiu-se um modelo sistemático de procedimentos que visam auxiliar os gestores a solucionar eventuais falhas em processos do setor de serviços, conforme Figura 3.

Figura 3 - Proposta de modelo de análise e melhoria de processos ao setor de serviços

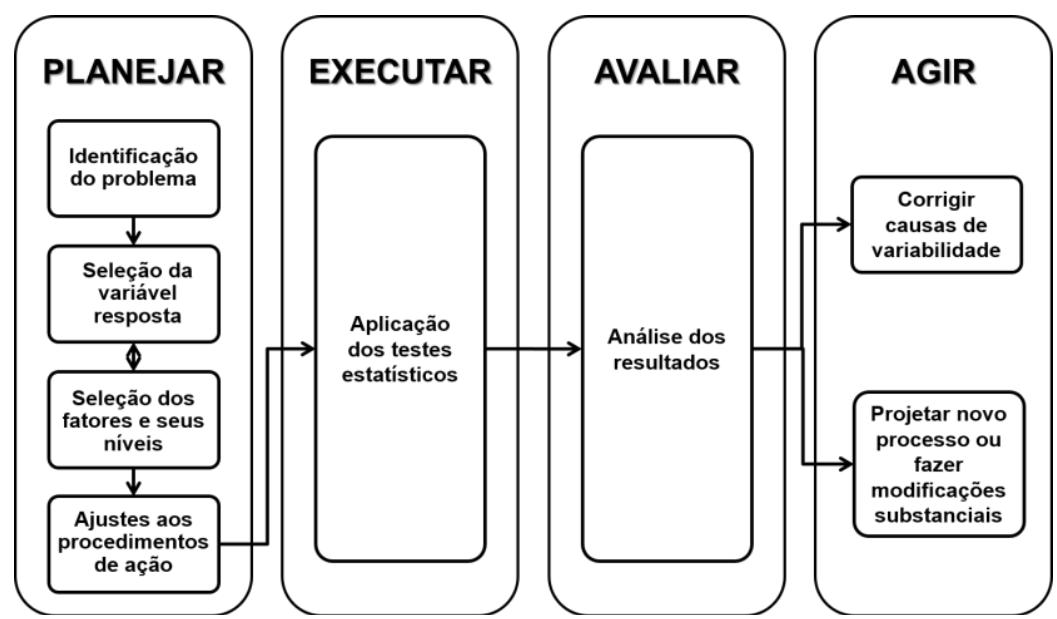

Fonte: Autoria própria (2013) 
Ressalta-se que esta união contempla tanto a análise descritiva dos processos quanto a análise estatística que assinala o aspecto científico. Em razão da metodologia do modelo proposto ser flexível, este modelo pode ser utilizado como referencial na melhoria de processos nas variadas áreas do setor de serviços.

Este modelo desdobra-se em quatro fases, descritas a seguir.

\section{PRIMEIRA FASE: PLANEJAR}

"O cerne de um programa de melhoria contínua consiste no pensamento de que não existe nada (nenhum processo) que não possa ser melhorado" (PEINADO; GRAEML, 2007, p. 558). Werkema e Aguiar (1996b) afirmam que esta é a fase mais complexa, contudo é a etapa fundamental para a realização da otimização de processos. Os autores afirmam ainda que nesta etapa são traçadas metas de melhorias e as ações que deverão ser executadas para se atingir as metas propostas. Johnson e Bell (2009) apontam que o planejamento cuidadoso inicial, através do esboço de projeto eficiente, com a escolha do tamanho de amostra suficiente e o profundo conhecimento dos processos em estudos são os itens mais importantes na pesquisa.

Primeiramente deve-se identificar o problema a ser corrigido, em geral, este problema é reconhecido pelos funcionários que possuem experiência e contato com os processos. "Definir o problema de forma consensual possibilita remover causas sem distorções" (DIAS, 2006, p. 50), assim este problema deve ser claro, objetivo e preciso, pois ele é a base para a execução do modelo.

Em seguida, através da observação e análise dos processos, deve-se selecionar apenas uma variável resposta ao estudo e os fatores que influenciam esta variável, sendo que a ordem desta seleção é irrelevante. Para viabilizar a execução do estudo, devem-se eleger os fatores chamados controláveis, isto é, aqueles que são possíveis de mensurar, como também se devem definir os níveis destes fatores. Gil (2002) nomeia este momento de construção das hipóteses, pois, nesta fase são estabelecidas relações causais entre os fatores interferentes do processo e a variável resposta, o autor sugere que estas relações sejam definidas pela fórmula "se... então".

Após essas escolhas, analisam-se os dados coletados, a fim de definir o plano de ação a ser executado, entende-se também esta fase como a etapa de ajustes aos procedimentos de ação. Neste momento são ponderados os dados coletados para a identificação da ferramenta estatística mais adequada a situação. Esta fase é crucial, considerando que sua má execução pode coletar dados poucos relevantes para pesquisa (WU; HAMADA, 2009).

\section{SEGUNDA FASE: EXECUTAR}

Esta fase consiste na execução do que foi traçado como procedimentos de ação na etapa passada, ou seja, neste momento são aplicados os testes estatísticos mais adequados para o conjunto de dados coletados, considerando suas características. Durante sua execução, devem-se registrar continuamente todos os resultados apresentados, através de folhas de verificação, por exemplo. 
Galdámez (2002) infere que com a aplicação desses testes uma série de questões relacionadas aos níveis e fatores que influenciam o desempenho do produto, seja bem ou serviço, são respondidas de modo a mitigar falhas no processo produtivo. Johnson e Bell (2009) afirmam que a mudança constante no mercado que passa a utilizar pessoas como unidades experimentais e fatores intangíveis faz requerer única atenção e estratégias diferentes e, às vezes, diferentes estatísticas.

Considerando o objetivo da pesquisa em propor um modelo de melhoria de processos baseado em técnicas estatísticas, sugere-se para esta fase as técnicas da Figura 4. Este esquema mostra algumas diferentes abordagens estatísticas que podem ser utilizadas em modelos experimentais, tanto para dados numéricos quanto para dados categóricos.

Figura 4 - Alguns procedimentos estatísticos para modelos experimentais

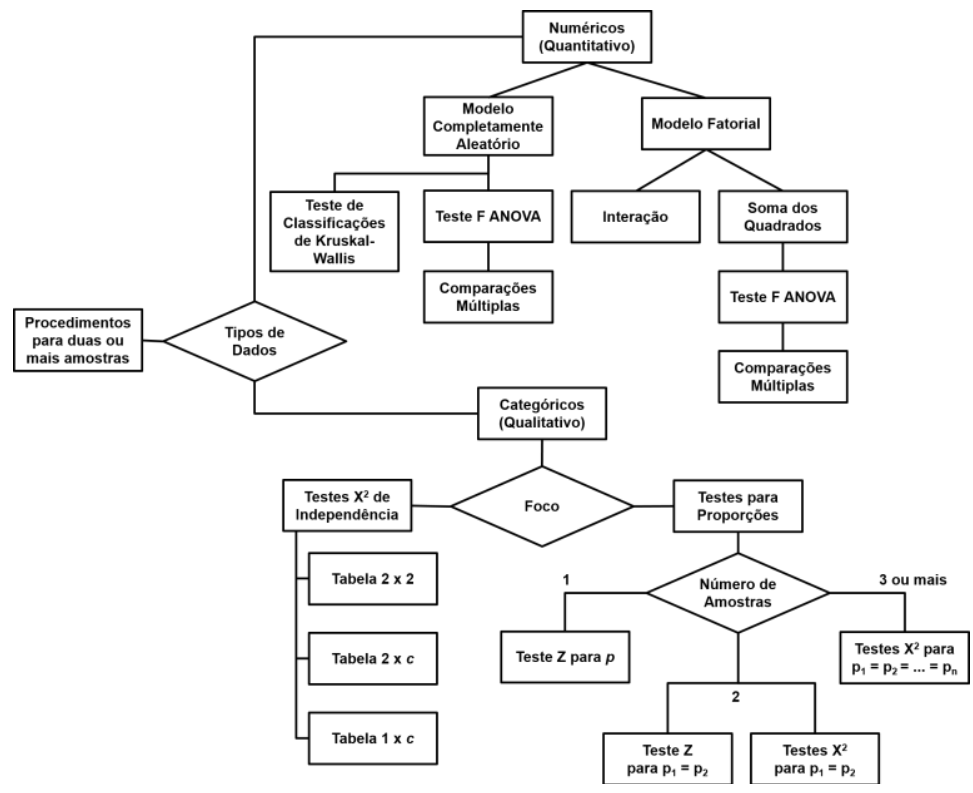

Fonte: Adaptado de Levine et al. (2005)

Ressalta-se que existem outras ferramentas derivadas das novas estruturas de produção que também são úteis na melhoria e otimização de processos produtivos e que poderiam ser utilizadas nesta fase do modelo, como: $5 \mathrm{~S}$, Kanban, Poka-yoke, Produção Enxuta (ou Lean Manufacturing), Kaisen, etc.

\section{TERCEIRA FASE: AVALIAR}

De posse dos resultados da etapa anterior, procede-se a revisão crítica dos resultados, a fim de interceptar possíveis erros de registro e omissões. Werkema e Aguiar (1996a) indicam a utilização de métodos gráficos para a representação dos dados e métodos estatísticos para se realizar a análise dos resultados. Wu e Hamada (2009) apontam que este resultado deve ser avaliado sob a luz do objetivo do estudo, ou seja, esta fase consiste em confirmar ou não a relação existente entre o problema (variável resposta) e a causa (fatores). Com base nestes resultados toma-se uma decisão para etapa futura, de forma que esta deliberação assegure a melhoria da qualidade dos processos. 
"O que precisamos fazer é encontrar as causas vitais dos produtos defeituosos e eliminar estas causas depois de terem sido identificados claramente" (KUME, 2002 apud RODRÍGUEZ; FRANCO, 2009, p. 154, tradução nossa). Considera-se esta etapa como a fase de tomada de decisão, onde se deve escolher a forma de atuação adequada para minimizar o problema identificado (FAN, 2013). Esta etapa consiste em atuar no processo em função da resposta da etapa anterior, são duas as possíveis formas de atuação:

a) corrigir causas da variabilidade do processo: tendo os testes determinado a condição ótima do processo e caso não haja grande discrepância, promove-se a melhoria da qualidade do processo, sendo que de acordo com Närman et al. (2012) o usufruto destas mudanças está condicionado a realizar manutenções permanentes. Esta melhoria pode ser feita através de treinamento da equipe de trabalho, melhoramento de condições de trabalho de forma a otimizar a utilização de equipamentos e ferramentas, como também pequenas modificações na modelagem do processo (WERKEMA; AGUIAR, 1996b);

b) implantar novo processo ou fazer modificações substanciais: no início são feitas mudanças em pequena escala que devem ser periodicamente monitoradas para verificar se os procedimentos estão sendo eficazes (DIAS, 2006), contudo Werkema e Aguiar (1996) ressaltam que na maioria das situações não é possível fazer grandes modificações, pois isto pode gerar grandes prejuízos para a empresa.

Caso ajam mudanças nos procedimentos de desenvolvimento dos processos, este novo procedimento adotado deve ser documentado como o objetivo de garantir que ele sempre seja utilizado a partir de então e até que uma nova melhoria o modifique, aconselha-se para isto a construção do Procedimento Operacional Padrão (POP).

Peinado e Graeml (2007) citam que os gerentes de produção sabem como é difícil implementar ação e romper a resistência natural das pessoas na organização, então eles apontam que uma boa estratégia é escolher a princípio, problemas fáceis de resolver, então, o sucesso servirá de treino e incentivo à equipe responsável.

Ressalta-se ainda, que para subsidiar estas fases, deve-se fazer uso das ferramentas da qualidade para a coleta e disposição dos dados, conforme apontado na Figura 5. 
Figura 5 - Ferramentas adequadas para as fases de planejar, checar e agir

\begin{tabular}{|c|c|c|c|c|c|c|c|c|}
\hline & \multirow[b]{2}{*}{ FERRAMENTAS } & \multicolumn{4}{|c|}{ FASES } & \multirow{2}{*}{ 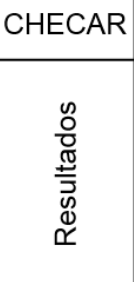 } & \multicolumn{2}{|c|}{ AGIR } \\
\hline & & $\begin{array}{l}\frac{\pi}{\tilde{E}} \\
\frac{0}{0} \\
\frac{0}{0} \\
\frac{0}{2}\end{array}$ & 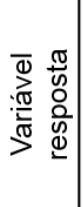 & 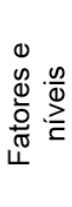 & 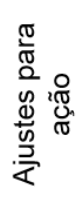 & & 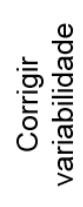 & 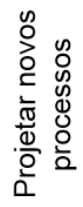 \\
\hline \multirow{7}{*}{ 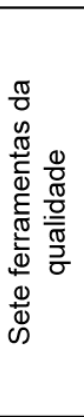 } & Estratificação & $\mathrm{X}$ & $\mathrm{X}$ & $\mathrm{X}$ & & $\mathrm{X}$ & $\mathrm{X}$ & \\
\hline & $\begin{array}{l}\text { Folha de } \\
\text { verificação }\end{array}$ & $x$ & $x$ & $x$ & & $x$ & $X$ & \\
\hline & Gráfico de Pareto & $x$ & $x$ & $x$ & & $x$ & & \\
\hline & $\begin{array}{l}\text { Diagrama de } \\
\text { causa-efeito }\end{array}$ & $x$ & $\mathrm{X}$ & $x$ & & & $\mathrm{x}$ & \\
\hline & Histograma & $\mathrm{X}$ & $\mathrm{X}$ & $\mathrm{X}$ & & $x$ & & \\
\hline & \begin{tabular}{|l|} 
Diagrama de \\
dispersão
\end{tabular} & & $x$ & $\mathrm{X}$ & & $x$ & & \\
\hline & $\begin{array}{l}\begin{array}{l}\text { Gráfico de } \\
\text { controle }\end{array} \\
\end{array}$ & $x$ & $\mathrm{X}$ & $\mathrm{X}$ & & $x$ & $x$ & \\
\hline \multirow{7}{*}{ 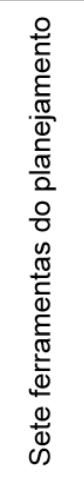 } & \begin{tabular}{|l|} 
Diagrama de \\
afinidades
\end{tabular} & $x$ & $x$ & $\mathrm{X}$ & & & & \\
\hline & \begin{tabular}{|l|} 
Diagrama de \\
relações
\end{tabular} & & & $\mathrm{X}$ & & & & \\
\hline & \begin{tabular}{|l|} 
Diagrama de \\
árvore
\end{tabular} & $x$ & X & $\mathrm{X}$ & & & & \\
\hline & $\begin{array}{l}\text { Diagrama de } \\
\text { matriz }\end{array}$ & $x$ & & $\mathrm{X}$ & & & & \\
\hline & \begin{tabular}{|l|} 
Diagrama de \\
priorização
\end{tabular} & $x$ & & $\mathrm{X}$ & & & & $\mathrm{x}$ \\
\hline & $\begin{array}{l}\text { Diagrama de } \\
\text { processo decisório }\end{array}$ & & & & & & & \\
\hline & Diagrama de setas & & & & & & & $x$ \\
\hline \multirow{7}{*}{ 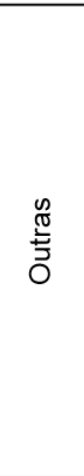 } & Brainstorming & $\mathrm{X}$ & $\mathrm{X}$ & $\mathrm{X}$ & & & $X$ & \\
\hline & $5 \mathrm{~W} 1 \mathrm{H}$ & & & & $\mathrm{X}$ & & $\mathrm{X}$ & $\mathrm{X}$ \\
\hline & \begin{tabular}{|l|} 
Operação \\
Evolutiva (EVOP) \\
\end{tabular} & & & & & & $\mathrm{X}$ & \\
\hline & $\begin{array}{l}\text { Experimento } \\
\text { fatorial } 2^{\mathrm{k}} \\
\end{array}$ & & & & & & & $x$ \\
\hline & $\begin{array}{l}\text { Metodologia de } \\
\text { superfície de } \\
\text { resposta } \\
\end{array}$ & & & & & & & $x$ \\
\hline & Benchmarking & & & & & & & \\
\hline & \begin{tabular}{|l} 
Controle \\
estatístico do \\
processo (CEP)
\end{tabular} & & & & & & $x$ & \\
\hline
\end{tabular}

Fonte: Adaptado de Araujo, Paula e Silva (2012), Carpinetti (2010), Gianesi e Corrêa (2011) e Werkema (1995).

\section{CONSIDERAÇÕES FINAIS}

Durante a revisão da literatura percebeu-se que a qualidade é importante para gerar clientes satisfeitos, neste viés instrumentos de mensuração do nível da qualidade são amplamente empregados nas mais diversas áreas do setor de serviço, inclusive no setor público; ainda assim tomar decisões com base em expectativas ou percepção dos clientes é algo impreciso, pois se reconhece que clientes com diferentes perfis têm diferentes visões sobre um mesmo produto ou serviço (SOUSA; YEUNG; CHENG, 2008). Sabendo-se que medidas objetivas são 
úteis para corrigir as avaliações fornecidas pelos usuários (PAQUETTE et al., 2012), toma-se esta pesquisa relevante por gerar dados objetivos para tornar a tomada de decisão mais eficiente, através da identificação da combinação ideal dos níveis dos fatores, o que viabiliza a otimização do processo.

Destaca-se que o modelo proposto segue os preceitos da atual Era da Qualidade (Gestão da Qualidade), a qual prega que a qualidade é gerada no processo, fato este que contrasta-se aos instrumentos comuns de qualidade, que 'medem' a qualidade ao fim do processo e não durante o processo produtivo. Salienta-se, também, que os alicerces do modelo foram feitos com metodologias consagradas pela literatura de gestão e cientifica, isto é, o Método de Análise e Solução de Problemas (MASP), derivado do Ciclo PDCA, e o Planejamento Experimental.

Sheu, Mchaney e Babbar (2003) ressaltam a necessidade de construir modelos flexíveis, pois segundo eles a otimização depende de vários parâmetros específicos do processo. Tendo sido o método escrito de forma genérica, se aceita que ele pode ser aplicado nas diversas áreas do setor de serviço, como: educacional, de saúde, bancário, administrativo, entre outros, isto tanto no campo privado como no público. E ainda que, o modelo seja proposto ao setor de serviços, admite-se também o seu emprego no setor de manufatura, isto permitiria avaliar a aplicabilidade do modelo em todos os tipos de processos produtivos, então, como desdobramento deste estudo, sugere-se a aplicação do modelo proposto nas áreas supramencionadas.

Durante a aplicação do modelo, devem-se quantificar os dados coletados para então aplicar o teste estatístico mais apropriado, caso os fatores trabalhados não sejam numéricos, pressupõem-se que haverá certa inquietação para sua quantificação, como solução a esta questão, recomenda-se utilizar a Escala Likert para quantificar o nível de concordância com o fator. Ressalta-se que a grande maioria dos fatores que causa interferência nos processos é do tipo qualitativo (categórico) (CARVALHO; BRITO; CABRAL, 2010; PAQUETTE et al., 2012), por exemplo: comunicação, competência, cortesia, credibilidade, confiabilidade, entre outros fatores específicos dos processos a serem analisados, talvez a dificuldade em quantificar este tipo de fator seja um dos motivos que torna escasso estudos semelhantes. 


\title{
Proposal for a model of analysis and optimization of processes for the service sector for the improvement of quality
}

\begin{abstract}
The literature points to several tools able to assess and improve service quality, however subjective mechanisms predominate, which boil down to measure customer satisfaction at the end of the process. Admittedly, the quality has focus on the customer, but in the current Era of Quality it is assumed that it is generated during the production process. Hence the objective of building a model of analysis and process improvement, backed in the precepts of quality management, able to optimize processes of service sector, through the use of management techniques and statistics, as well as generate objective data so that the decision-making process was made more robust. For this, developed a theoretical-conceptual research, qualitative aspect and basic nature. At the end, through the link of the phases of the Method for Analysis and Solution of Problems (MASP) with the steps of Design of experiments the model has been obtained.
\end{abstract}

KEYWORDS: Model. Quality. Process. Service. 


\section{REFERÊNCIAS}

BALLOU, R. H. Logística empresarial: transportes, administração de materiais, distribuição física. São Paulo: Atlas, 2007.

ANTONY, J.; COLEMAN, S.; MONTGOMERY, D. C.; ANDERSON, M. J.; SILVESTRINI, R. T. Design of experiments for non-manufacturing processes: benefits, challenges and some examples. Proceedings of the Institution of Mechanical Engineers, Part B: Journal of Engineering Manufacture. United Kingdom. v. 225, n. 11, p 2078-2087, 2010. D.O.I.: $10.1177 / 0954405411395857$ crossref

ARAUJO, L. B.; PAULA, I. A. A; SILVA, O. C.. Estudo sobre uso do Ciclo PDCA na gestão da qualidade de processos no setor de serviços. In.: SIMPÓSIO DE ENGENHARIA DE PRODUÇÃO, 19., 2012, Bauru. Anais... Bauru: Faculdade de Engenharia Bauru, 2012. p. 112. ISSN 1809-7189.

BATALHA, M. O. (organizador). Introdução à engenharia de produção. Rio de Janeiro: Elsevier, 2008.

CARPINETTI, L. C. R. Gestão de qualidade: conceitos e técnicas. São Paulo: Atlas, 2010.

CARVALHO, C.; BRITO, C.; Cabral; J. S. Towards a conceptual model for assessing the quality of public services. International Review on Public and Nonprofit Marketing. New York. V. 07, N. 01, P. 69-86, 2010. DOI 10.1007/s12208-010-0046-5.

CERVO, A. L.; BERVIAN, P. A.; SILVA, R. Metodologia Científica. 6. ed. São Paulo: Pearson Prenticce Hall, 2007.

COLLIER, D. A.; MEYER, S. M. A service positioning matrix. International Journal of Operations \& Production Management, United Kingdom, v. 18, n. 12, p. 1223-1244, 1998. DOI: $10.1108 / 01443579810236647$. cross ref

CORRÊA, H. L.; CORRÊA, C. A. Administração de produção e operações - manufatura e serviços: uma abordagem estratégica. São Paulo: Atlas, 2004.

DIAS, E. E. P. Análise de Metodologia de Melhoria de Processos: Aplicações à Indústria Automobilística. 2006. 100 f. Dissertação (Mestrado em Sistemas de Gestão) Universidade Federal Fluminense, Niterói, 2006.

FAN, X.-Q. A decision-making method for personalized composite service. Expert Systems with Applications. Expert Systems with Applications: An International Journal, Philadelphia, v. 40, n. 15, p. 5804-5810. 2013. DOI: 10.1016/j.eswa.2013.05.018. crossref

FORNO, N. L. F. D. Clima Organizacional e Qualidade em Serviços: Estudo de caso em laboratório de análises clínicas. 2005. 180 f. Dissertação (Mestrado em Engenharia de Produção) - Universidade Federal de Santa Maria, Rio Grande do Sul, 2005.

GALDÁMEZ, E. V. C. Aplicação das técnicas de planejamento e análise de experimentos na melhoria da qualidade de um processo de fabricação de produtos plástico. 2002. 133 f. Dissertação (Mestrado em Engenharia de Produção) - Escola de Engenharia de São Carlos, Universidade de São Paulo, São Paulo, 2002. 
GIANESI, I. G. N.; CORRÊA, H. L. Administração estratégica de serviços: operações para a satisfação do cliente. 1. ed. 21. São Paulo: Atlas, 2011.

GIL, A. C. Como Elaborar Projetos de Pesquisa. 4. ed. São Paulo: Atlas, 2002.

JOHNSON, Lou; BELL, Gordon. Designed experiments in service quality applications. In: WORLD CONGRESS ON QUALITY AND IMPROVEMENT, 2009. Anais... ASQ Conference. 2009. p. 18-20.

JUNG, C. F. Elaboração de projetos de pesquisa aplicados a engenharia de produção. Taquara: FACCAT, 2010. Disponível em: <http://www.metodologia.net.br>. Acesso em: 28 mar. 2012.

JUNYENT, I.; VILLALBÍ, J. R.; BALLESTÍN, M.; DURÁN, J.; GARCÍA-RODRÍGUEZ, A.; PERACHO, V. Evaluación de la calidad en salud pública: aplicación a un centro de acogida de animales de compañía. Gaceta Sanitaria. Espanã. v. 23, n. 05, p. 440-443, 2009. D.O.I.: 10.1016/j.gaceta.2009.02.009

LEVINE, David M. et al. Estatística - Teoria e Aplicações usando o Microsoft ${ }^{\circledR}$ Excel em $^{-}$ Português. 3. ed. Rio de Janeiro: LTC Editora, 2005.

LIMA, L. G. G. B. Planejamento de Experimentos Bayesianos: Aplicações em Experimentos na Presença de Tendências Lineares. 2006. 121 f. Dissertação (Mestrado em Estatística) Escola de Engenharia de São Carlos, Universidade de São Paulo, São Paulo, 2006.

MIGUEL, P. A. C. (Org.). Metodologia de pesquisa em engenharia de produção e gestão de operações. Rio de Janeiro: Elsevier, 2010.

MONTGOMERY, D. C. Introdução ao controle estatístico da qualidade. Tradução Ana Maria Lima de Farias, Vera Regina Lima de Farias e Flores. Revisão técnica: Luiz da Costa Laurencel. 4 ed. Rio de Janeiro: LTC, 2009.

NÄRMAN, P.; HOLM, H.; EKSTEDT, M.; HONETH, N. Using enterprise architecture analysis and interview data to estimate service response time. Journal of Strategic Information Systems, Philadelphia, v. 22, n. 3, p. 70-85, 2012. DOI: 10.1016/j.jsis.2012.10.002. crossref

PALADINI, E. P. Gestão da qualidade no processo: a qualidade na produção de bens e serviços. São Paulo: Atlas, 1995.

PAULA, I. A. A.; SILVA, O. C. Otimização de processos no Setor de Serviço para a melhoria da Qualidade: Experimento em um Instituto Federal de Ensino. Produção em Foco, [S.I.], v. 6, n. 1, mar. 2016. ISSN 2237-5163. Disponível em: <http://producaoemfoco.org/index.php/producaoemfoco/article/view/209>. Acesso em: 28 set. 2017.

PAQUETTE, J.; BELLAVANCE, F.; CORDEAU, J. F.; LAPORTE, G. Measuring quality of service in dial-a-ride operations: the case of a Canadian city. Transportation, New York, v. 39, n.3, p. 539-564. 2012. DOI: 10.1007/s11116-011-9375-4. crossref

PARASURAMAN, A.; ZEITHAML, V. A.; BERRY, L. L. A concep tual model of service quality and its implications for future research. Journal of Marketing. USA. v. 49, n. 4, p. 41-50, fall 1985.

PEINADO, J.; GRAEML, A. R. Administração da produção: operações industriais e de Serviço. Curitiba: UnicenP, 2007. 
PRASS, R. M.; SANT'ANNA, L. C.; GODOY, L. P. Avaliação da Qualidade de Serviços Prestados na área educacional através do modelo SERVQUAL. Revista Gestão Industrial. Ponta Grossa, v. 06, n. 02, p. 213-231, 2010. D.O.I.: 10.3895/S1808-04482010000200012.

RAMSEOOK-MUNHURRUN, P.; LUKEA-BHIWAJEE, S. D.; NAIDOO, P. Service Quality in the Public Service. International Journal of Management and Marketing Research. Austria. v. 03, n. 01, p. 37-50, 2010.

RIBEIRO, M. N. A. M. Apoio ao domicílio: impacto e qualidade percebida pelo cliente. 2010. 66 f. Dissertação (Mestrado em Gestão) - Departamento de Economia, Gestão e Engenharia Industrial, Universidade de Aveiro, Aveiro, 2010.

RODRÍGUEZ, A. I. P.; FRANCO, J. R. Control estadístico de la calidad de un servicio mediante Gráficas X y R. Política y cultura. México. n. 32, p. 151-169, 2009.

SHEU, C.; MCHANEY, R.; BABBAR, S. Service process design flexibility and customer waiting time. International Journal of Operations \& Production Management, United Kingdom, v. 23, n. 8, p. 901-917, 2003. DOI: 10.1108/01443570310486347. crossref

SILVA, E. L.; MENEZES, E. M. Metodologia da pesquisa e elaboração de dissertação. 4. ed. rev. atual. Florianópolis: UFSC, 2005.

SIQUEIRA, D. M. R. Avaliação da qualidade em serviços: uma proposta metodológica. 2006. 213 f. Tese (Doutorado em Engenharia de Produção) - Universidade Federal de Santa Catarina, Florianópolis, 2006.

SOUSA, T. C. G. D.; SILVEIRA, A.; FORTES, V. C.; DOMINGUES, M. Comparação de modelos de qualidade de serviço: proposição estratégica para instituições de ensino superior. In.: SEMINÁRIOS EM ADMINISTRAÇÃO, 14., 2011, São Paulo. Anais... SãoPaulo: Faculdade de Economia, Administração e Contabilidade da Universidade de São Paulo, 2011. p. 1-16. ISSN 2177-3866.

SOUSA, R.; YEUNG, A. C. L.; CHENG, T. C. E. Customer heterogeneity in operational eservice design attributes: an empirical investigation of service quality. International Journal of Operations \& Production Management, United Kingdom, v. 28, n. 7, p. 592614. 2008. DOI: 10.1108/01443570810881776. crossref

VALLS, V. M. Gestão da qualidade em serviços de informação no Brasil: Estabelecimento de um modelo de referência baseado nas diretrizes da NBR ISSO 9001. 2005. 256 f. Tese (Doutorado em Ciências da Comunicação) - Universidade de São Paulo, São Paulo, 2005.

WERKEMA, M. C. C. As ferramentas da qualidade no gerenciamento de processos. Belo Horizonte: Fundação Christiano Ottoni, Escola de Engenharia da UFMG, 1995.

WERKEMA, M. C. C; AGUIAR, S. Planejamento e análise de experimentos: como identificar a avaliar as principais variáveis influentes em um processo. Belo Horizonte: Fundação Christiano Ottoni, Escola de Engenharia da UFMG, 1996a.

WERKEMA, M. C. C; AGUIAR, S. Otimização estatística de processos: como determinar a condição de operação de um processo que leva ao alcance de uma meta de melhoria. Belo Horizonte: Fundação Christiano Ottoni, Escola de Engenharia da UFMG, 1996b.

WU, C. F. J.; HAMADA, M. S. Research design: Qualitative, quantitative, and mixed methods approaches. 2. ed. United Stat. 
Recebido: 28 set. 2017

Aprovado: 16 abr. 2018

DOI: 10.3895/gi.v14n2.7124

Como citar:

PAULA, I. A. A. Proposta de modelo de análise e otimização de processos para o setor de serviços visando a melhoria da qualidade. R. Gest. Industr., Ponta Grossa, v. 14, n. 2, p. 227-244, abr./jun. 2018. Disponível em: https://periodicos.utfpr.edu.br/rgi $>$. Acesso em: XXX.

Correspondência:

Izabel Alinne Alves de Paula

Rua Jonathas Pedrosa, n. 023, Bairro: Praça 14 de Janeiro, CEP: 69020-110, Manaus, Amazonas, Brasil. Direito autoral:Este artigo está licenciado sob os termos da Licença Creative Commons-Atribuição 4.0

Internacional.

(c) (1) 